\title{
CollabEduc: Uma Ferramenta de Colaboração em Pequenos Grupos para Plataformas de EaD
}

\author{
Edviges de Fátima Chaves de Lima ${ }^{1}$, Clauirton de Albuquerque Siebra ${ }^{1}$ \\ ${ }^{1}$ Centro de Informática - Universidade Federal da Paraíba (UFPB) \\ - João Pessoa - PB - Brasil \\ \{edvigeslima, clauirton\}@ci.ufpb.br
}

\begin{abstract}
Distance Learning (DL) comes as an alternative to meet the needs of students who look for a higher education degree, but live in remote regions and cannot travel to capitals or big cities, by lacking in time or money. Unfortunately, the high dropout and retention rates show that the use of DL in countries, such as Brazil, is far from the expected results. In this way, after the search and analysis of several theories/methods on collaborative learning, three approaches were chosen to be evaluated, which help to improve this reality. Then, such approaches were used as basis to a computational resource, called CollabEduc, whose main aim is to motivate collaboration among participants.
\end{abstract}

Resumo. A Educação a Distância (EaD) é uma alternativa para estudantes que almejam uma formação superior, mas vivem em regiões distantes e não tem condições de se deslocar para os grandes centros, seja por limitações de tempo elou financeiras. Infelizmente, as altas taxas de evasão e retenção mostram que o uso da EaD ainda está longe dos resultados esperados. Dessa forma, após o estudo de teorias/métodos colaborativos, foram definidas três estratégias que possam ajudar a melhorar essa realidade. Tais estratégias foram utilizadas como base para a implementação de um recurso computacional chamado CollabEduc, cujo principal objetivo é motivar a colaboração entre participantes ao longo de atividades educacionais.

\section{Introdução}

A Educação a Distância $(\mathrm{EaD})$ tem um grande potencial para o sucesso porque ela oferece uma atraente relação custo-benefício para alunos e instituições, uma vez que tais instituições se tornam capazes de atrair um maior número de alunos a um custo razoável (Maia, 2008). Por outro lado, do ponto de vista educacional de Sherry (1995), o que parece ser inovador e radical também apresenta muitos problemas, tais como falta de interatividade e falta de estímulo aos alunos. O censo ABED (2014) dispõe ainda que, em geral, os alunos não são capazes de se adaptar à rotina de estudos individuais que esta modalidade de ensino requer, alegando falta de tempo para estudar e participar do curso. Assim, eles recebem notas baixas durante as avaliações, acabam perdendo a motivação e abandonando seus cursos. Isso ocorre principalmente durante o primeiro semestre de estudos, onde é observada a maior taxa de abandono.

Vários estudos educacionais e psicológicos indicam que as interações melhoram a aprendizagem (Dillenbourg et al., 1996). Tais estudos se apoiam, por exemplo, na ideia proposta por Blom, Verma, et al. (2013) que discute que os pares de alunos podem encontrar apoio um no outro para resolver as tarefas. Neste contexto também é 
considerado o formato social como sendo natural uma vez que os alunos já estão acostumados com a ideia de se engajar em práticas de grupo de estudo, conforme praticado no ensino tradicional. O trabalho apresentado por Chiong e Jovanovic (2012) é outro exemplo. Eles propõem uma abordagem pragmática para promover a aprendizagem colaborativa em pequenos grupos através do mapeamento de estratégias educacionais de aprendizagem colaborativa em recursos computacionais que possam ser aplicados dentro de ambientes virtuais de ensino. Com isso, pretendeu-se proporcionar uma melhor interação/comunicação entre os alunos, aumento da capacidade de aquisição de conhecimento e, por consequência, uma melhor aprendizagem.

O presente trabalho segue essa linha e também busca um maior suporte para a colaboração entre alunos. Para isso, nossa proposta está estruturada da seguinte forma. A seção 2 apresenta as teorias de aprendizagem que suportam nosso modelo computacional. A seção 3 descreve as ferramentas e os métodos utilizados na elaboração do projeto. Na seção 4 é apresentado o experimento, bem como os resultados obtidos. Por fim, a seção 5 discute as considerações finais e os trabalhos futuros.

\section{Teorias da Aprendizagem com Suporte à Colaboração}

Em geral, as teorias da aprendizagem têm por objetivo distinguir quais as condições essenciais e mais apropriadas para que a aprendizagem ocorra no indivíduo. Desta forma, as seguintes Teorias de Aprendizagem foram utilizadas na formação das estratégias de suporte à colaboração do nosso trabalho.

A Teoria de Piaget (Piaget, 1976) (Piaget, 1977) dispõe a ideia de que a inteligência do indivíduo é uma constante adaptação. A construção do conhecimento ocorre a partir de suas descobertas quando em contato com o mundo e com os objetos. Aplicada à educação, essa teoria propõe colocar os estudantes em atividades reais e desafiadoras na comunidade de aprendizagem. Ou seja, através da prática colaborativa para solucionar problemas, cada indivíduo do grupo terá uma perspectiva diferente do problema e irá interagir, explanar e discutir com os outros membros buscando uma compreensão compartilhada.

Vygotsky (1979) dispõe em sua teoria que o convívio e as interações sociais são uns dos fatores fundamentais para a aprendizagem. Ele postula ainda que $o$ conhecimento é definido através do discernimento do que indivíduo é capaz de fazer sem ajuda e o que ele pode fazer interagindo com o meio social. Em termos educacionais, a aprendizagem é o meio pelo qual o estudante adquire conhecimento, desenvoltura, valores, etc. mediado e regulado por suas relações reais com o meio social e as condições histórico-culturais em que o mesmo está inserido. Assim, pode-se considerar que a utilização de recursos como trabalhos em grupo, o uso de tecnologias comunicativas como bate-papos, fóruns de discussão e outras formas de comunicação em grupos, podem levar ao debate de diferentes ideias e ao desencadeamento de novos conflitos cognitivos.

Por fim, Siemens (2004) defende a teoria de que os princípios e processos de aprendizagem são reflexos do contexto atual dos indivíduos. Em contrapartida às teorias anteriormente citadas, a teoria de Siemens busca reconhecer as possibilidades pedagógicas trazidas pela tecnologia, apoiando a aprendizagem e o conhecimento na diversidade de opiniões, onde o ponto de partida da aprendizagem é o indivíduo que 
VI Congresso Brasileiro de Informática na Educação (CBIE 2017)

Anais do XXVIII Simpósio Brasileiro de Informática na Educação (SBIE 2017)

alimenta informações para a rede, que alimenta informações de volta para os indivíduos que, por sua vez alimentam as informações de volta para a rede como parte de um ciclo.

\section{Ferramentas e Métodos}

\subsection{Modelagem das Estratégias de Suporte à Colaboração}

Para a modelagem das estratégias de suporte à colaboração, foi utilizada a Grounded Theory (GT) ou Teoria Fundamentada em Dados (TFD), a qual é composta dos seguintes passos: etapa de comparação dos dados coletados (análise); identificação de palavras-chave a fim de sintetizar informações (codificação); extração e agrupamento das similaridades destas informações fomentando conceitos (conceptualização), e agrupamento de conceitos semelhantes que podem emergir em algumas teorias (categorização) (Charmaz, 2006). Conforme disposto por Ferreira e Felizola (2012), "a estratégia proposta pela GT é trabalhar com uma amostragem teórica onde o pesquisador recolhe, codifica e analisa informações de fontes que provavelmente fornecerão dados relevantes". Dessa forma, a base para a coleta de dados ficou delimitada aos trabalhos da literatura que tratem das etapas de construção de conhecimento, bem como, discussões sobre motivação e colaboração com o objetivo de aperfeiçoar o processo de aprendizagem.

$\mathrm{Na}$ etapa de codificação, ou codificação inicial, devem-se extrair algumas palavras-chave (ou codes) que serão a matéria-prima para a formação dos conceitos. $\mathrm{O}$ presente trabalho tomou como ponto de partida os textos das teorias de aprendizagem

aqui apresentadas. Seguindo para a etapa de conceptualização, seguiu-se o modelo sistemático onde as palavras-chave são convertidas, de modo empírico, em conceitos (ou concepts). Por se tratar de uma etapa intermediária entre a codificação e o primeiro rascunho da análise concluída, a mesma foi baseada em observações e insights. Dessa forma, alinhados aos conceitos propostos por Dillenbourg e Hong (2008), foram fomentados os termos: argumentação, explanação e regulação mútua.

\subsection{Ferramenta CollabEduc}

Após o estudo das teorias de aprendizagem colaborativa e a definição das três estratégias de suporte a colaboração, surgiu a necessidade de implementar algo onde fosse possível testar a efetividade das mesmas. As plataformas de EaD possuem uma série de recursos que fornecem aos estudantes diferentes alternativas para conteúdo e atividades educacionais. Então, tomando isso como base, surge a ferramenta chamada CollabEduc, que tem por objetivo dar suporte a pequenos grupos de estudo através da aplicação das estratégias discutidas na subseção anterior.

Em seu trabalho, Blom, Verma, et al. (2013) propõem o seguinte cenário: "Imagine uma sala de aula, onde um estudante interrompe o professor e diz: - Desculpe, senhor. Você poderia fazer uma pausa de três minutos enquanto eu discuto com meu colega o que acabou de dizer?". Este cenário dificilmente ocorreria em aulas normais, mas ocorre espontaneamente quando se assiste gravações em vídeo destas mesmas aulas. Este cenário é uma forma interessante para fornecer colaboração e, tal como salientado por Blom, Verma, et al. (2013), é fácil de ser implementado através de vídeos. O esquema da Figura 1 apresenta uma possibilidade de recurso para colaboração em pequenos grupos, o qual está servindo de base para o CollabEduc. 
VI Congresso Brasileiro de Informática na Educação (CBIE 2017)

Anais do XXVIII Simpósio Brasileiro de Informática na Educação (SBIE 2017)

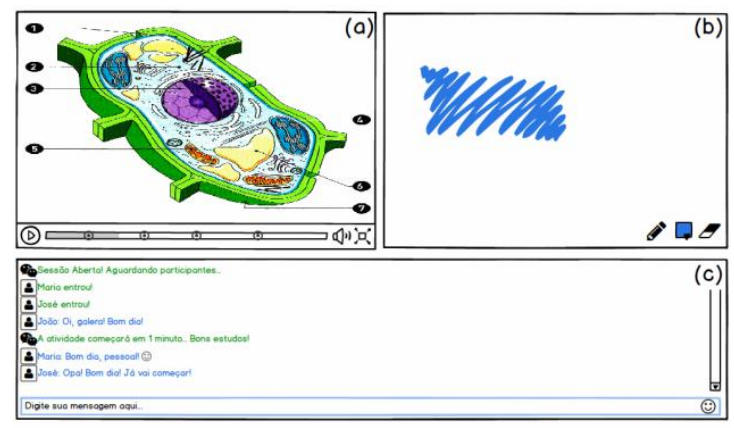

Figura 1. Esquema do Recurso Educacional para Colaboração em Pequenos Grupos. (a) Player de Vídeo, (b) Quadro Branco Virtual, (c) Chat

Este esquema inicial para um recurso de colaboração em pequenos grupos é formado por três partes. Sejam elas:

- Player de vídeo (Figura 1a): através deste componente, os membros do grupo terão acesso às videoaulas dos assuntos que servirão de base para interação e comunicação entre os membros do grupo. Nele, é possível inserir várias pausas estratégicas que trarão questionamentos sobre o assunto da videoaula onde as mesmas serão respondidas de forma colaborativa pelo grupo, de acordo com o método de suporte à colaboração selecionado;

- Quadro branco virtual (Figura 1b): este componente será responsável por apoiar as discussões entre os membros. Mellingsæter (2014) discute que o uso de quadro branco virtual tem o potencial para melhorar a coletividade dos estudantes, permitindo que cada membro do grupo tenha acesso à solução do problema a qualquer instante. Dessa forma, a construção da solução do problema em conjunto permite uma melhora significativa no desempenho do estudante;

- Chat (Figura 4c): neste componente, os estudantes poderão interagir através de mensagens de texto e, assim, discutir entre si possíveis soluções para os questionamentos expostos nas pausas estratégicas do vídeo.

\subsection{Estratégias de Colaboração na Ferramenta CollabEduc}

Com o objetivo de perturbar a convergência natural dos seus utilizadores e proporcionar uma melhor aprendizagem, foram delimitadas especificações de objetos de aprendizagem para colaboração em pequenos grupos considerando as três estratégias de colaboração concebidas no início do trabalho. São elas: argumentação, explanação e regulação mútua.

\subsubsection{Argumentação}

$\mathrm{O}$ ato de argumentar não deve ser uma característica de cunho exclusivo a filósofos e cientistas. Trata-se de um comportamento útil para a vida cotidiana e profissional de qualquer pessoa. $\mathrm{O}$ argumento é importante porque ensaiam na vida pública os vários lados de um problema ou um ponto de disputa. É a primeira fase de solução de um problema. No contexto educacional, a argumentação tem evoluído de forma crescente, não apenas por ser uma competência importante a ser aprendida, mas também porque a argumentação pode ser utilizada para promover aprendizado em diversos domínios (Luca e Kubo, 2011) (Muller Mirza e Perret-Clermont, 2009) (Andrews, 2009) (Costa, 2008). 
A estratégia de argumentação, conforme esquema da Figura 2, pode ser inserida neste contexto com o envio de questionamentos sobre o tema aos estudantes em pausas estratégicas do vídeo. Após a submissão das respostas, se verificada uma divergência entre os estudantes, os mesmos serão convidados a arguir até chegar a um consenso. Após algum tempo, outro questionamento de igual teor será enviado aos estudantes. Se as divergências permanecerem, o ciclo é repetido por um número limitado de vezes.

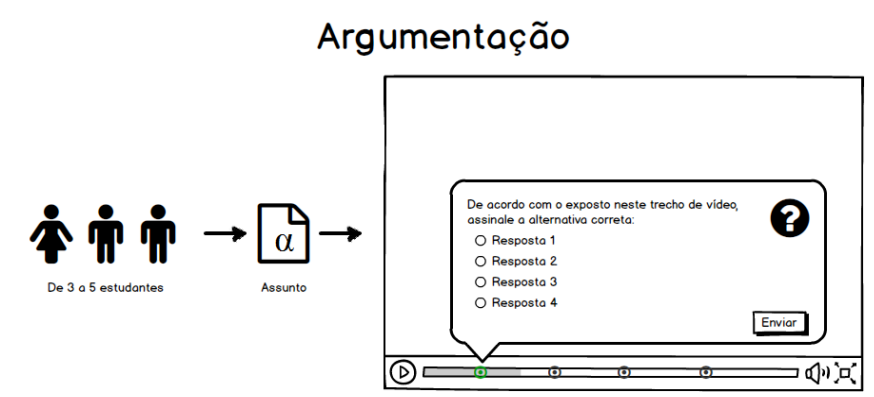

Figura 2. Exemplo Prático da Aplicação do Conceito de Argumentação.

\subsubsection{Explanação}

Uma alternativa aos métodos de ensino em salas de aula convencionais, a técnica Jigsaw (Aronson, Blaney, et al., 1978), também chamada de "quebra-cabeças", tem recebido resultados positivos em relação à aprendizagem. Em vez de agrupar toda uma classe em torno de um professor, os estudantes são ensinados a trabalhar em grupos menores interdependentes; cada estudante recebe uma parte de um tópico a ser estudado e, quando terminar, os estudantes explicam sua parte a fim de formar o "quebra-cabeça" completo com o assunto. Assim, diferentemente do cenário anterior, os estudantes irão assistir a vídeos diferentes. No final dos vídeos, a atividade final é apresentada para o grupo e então os estudantes precisarão interagir e unir conhecimento para conseguir resolver a atividade. Este esquema está ilustrado na Figura 3.

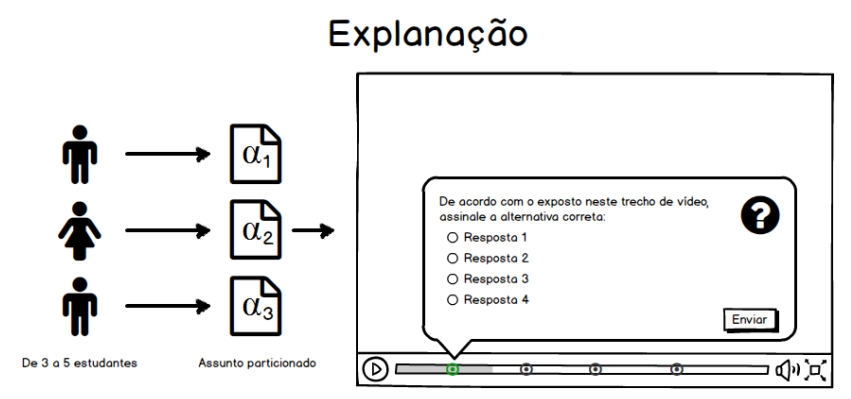

Figura 3. Exemplo de Conhecimento Fragmentado Usando Vídeos Interativos.

\subsubsection{Regulação Mútua}

A regulação mútua analisa a forma como os indivíduos reciprocamente regulam os processos cognitivos uns dos outros e envolvem-se em modos comuns de regulação cognitiva. Os objetos de aprendizagem que permitem a criação conjunta de uma solução por estudantes que contribuem mutuamente uns com os outros podem fomentar a regulação mútua. $\mathrm{O}$ esquema da Figura 4 mostra um possível cenário onde pode ser configurado um recurso educacional para colaboração em pequenos grupos fazendo uso do quadro branco virtual. Após a exibição da videoaula, uma (ou várias) questões são ativas e os estudantes são estimulados a construir a solução dessa atividade no quadro branco virtual. 
Nesta abordagem, cada estudante do grupo tem um tempo pré-definido para contribuir para a solução. Enquanto isso, os outros estudantes observam tal construção. Passado o tempo estipulado, a construção é pausada e a vez passa para o próximo estudante. Todos os estudantes devem manter a atenção na resolução do grupo, uma vez que cada um deles irá retomar a construção do ponto onde foi interrompida. Essa abordagem também instiga o estudante a pensar à frente, de acordo com a linha contínua de resolução, para que eles possam ter um melhor desempenho em sua vez.

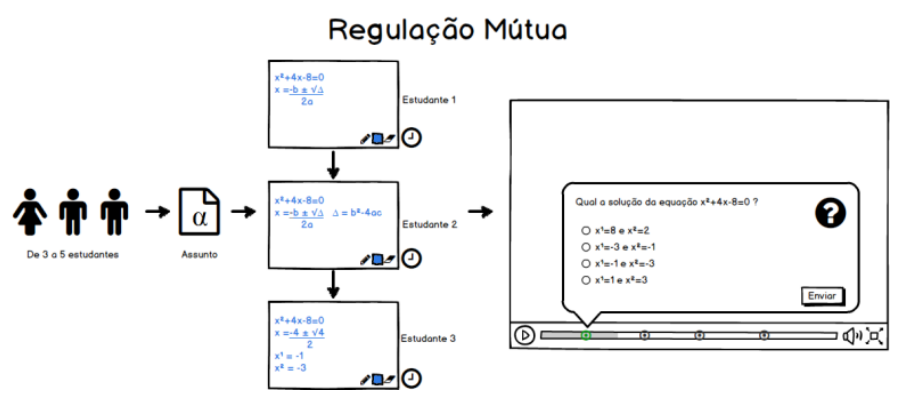

Figura 4. Esquema de Regulação Mútua.

\section{Experimento e Resultados}

A fim de validar as estratégias antes de efetivamente aplicá-las ao CollabEduc, utilizouse uma versão simplificada da metodologia de validação proposta por Costa Segundo (2011). A mesma foi dividida em três partes: elaboração, execução e análise/ consolidação dos resultados.

\subsection{Elaboração e Execução da Validação}

Após a definição dos parâmetros e métodos a serem utilizados na validação, a fase de elaboração compreendeu atividades tais como definição do público-alvo, definição dos parâmetros comparativos, especificação dos testes, elaboração dos questionários, elaboração do relatório dos monitores. Na fase de execução, por sua vez, objetivou-se o recrutamento de turmas, aplicação de questionário sobre o entendimento dos participantes a respeito da formação de grupos de estudo, bem como, utilização de ferramentas de apoio à motivação e colaboração, divisão de grupos (controle e teste), aplicação das estratégias de colaboração e questionário qualitativo com o grupo de teste e, por fim, aplicação de uma avaliação final do experimento com todos os participantes.

A validação das estratégias de colaboração consistiu no recrutamento de turmas do Ensino Superior que possuíssem conhecimentos mínimos sobre algoritmos e programação. Ao todo, o experimento, ocorrido em agosto do ano de 2016, contou com a presença de 134 participantes divididos em 4 turmas. Cada uma das turmas foi dividida em dois grandes grupos (teste e controle) e, no grupo de teste, os participantes foram subdivididos em grupos menores de 4 a 5 participantes, pois, conforme proposto por Cunha e Siebra (2015), a preferência por grupos menores evita a presença de membros não atuantes que irão explorar os esforços de membros atuantes gerando insatisfação e desmotivação. Cada subgrupo, acompanhado por um monitor (alunos regulares do programa de Pós-graduação em Informática da UFPB), recebeu uma lista de exercícios com 3 questões sobre um assunto pré-definido. Definiu-se ainda que os participantes poderiam fazer uso de qualquer material de apoio. 
Para cada questão aplicada aos participantes foi protocolada uma das estratégias de colaboração (argumentação, explanação ou regulação mútua). Todo o processo foi cuidadosamente observado pelo monitor que, por sua vez, relatou posteriormente todos os fenômenos ocorridos durante o experimento. Após a aplicação de um questionário qualitativo sobre o uso das estratégias no experimento, foi aplicada uma avaliação com questões simples para os dois grandes grupos (teste e controle), sem o uso de material de apoio, com o intuito de verificar o nível da turma. Salienta-se ainda que, por não se tratar de uma validação com corte longitudinal, ou seja, que acompanha o desenvolvimento do processo de aprendizagem do início ao fim do período letivo, não foi possível inferir se houve evolução na aprendizagem dos participantes.

\subsection{Análise e Consolidação dos Resultados}

Após a fase de execução do experimento, iniciou-se a fase de consolidação dos resultados a partir da análise dos dados obtidos no questionário sobre o entendimento de todos os participantes em relação ao conceito geral de formação de grupos de estudos e suas respectivas expectativas acerca da utilização desse conceito de uma forma virtualizada (Figura 5).

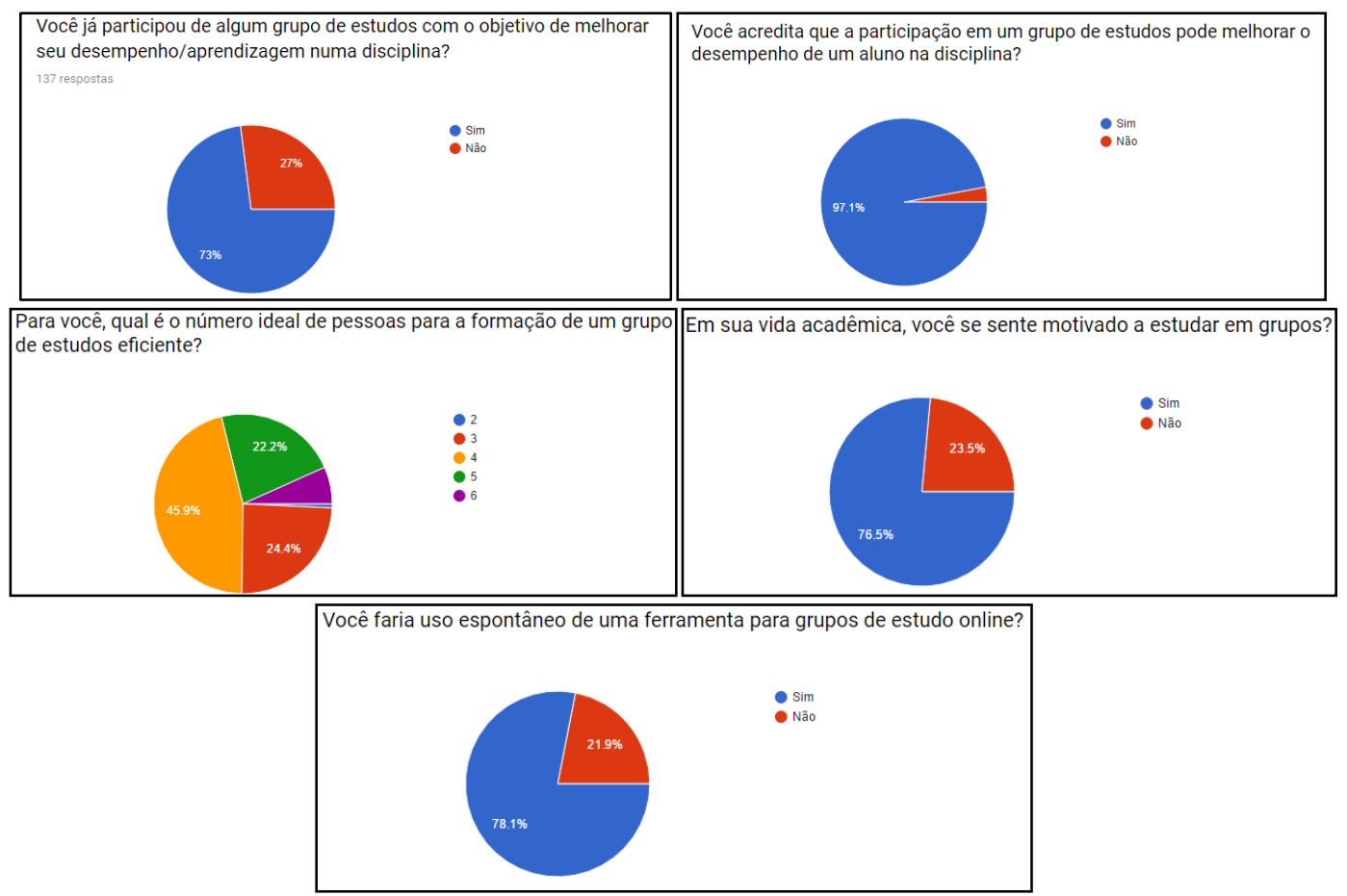

Figura 5. Gráficos resultantes dos questionários sobre o entendimento dos participantes.

Conforme pode ser observado na Figura 5, os participantes foram receptivos ao conceito de grupos de estudos, bem como sobre a utilização de uma ferramenta para grupos de estudos online. A partir dos dados coletados das observações dos monitores, na Tabela 1, temos o desempenho geral dos Grupos de Teste dentro de cada uma das 4 turmas avaliadas na estratégia de Argumentação. Nela, é possível observar que 53,32\% discordaram das respostas após a primeira tentativa para resolver a questão e, a maioria $(89,6 \%)$ colaborou para chegar a um consenso. 
VI Congresso Brasileiro de Informática na Educação (CBIE 2017)

Anais do XXVIII Simpósio Brasileiro de Informática na Educação (SBIE 2017)

Já na estratégia de Explanação, ainda conforme a Tabela 1 à direita, pode-se observar que $98,3 \%$ dos participantes interagiram e colaboraram na busca pela resolução da questão. No entanto, o tempo pré-determinado para explanação mostrou-se insuficiente dada a complexidade da questão.

Tabela 1: Resultados obtidos com a Argumentação (esq.) e a Explanação (dir.).

\begin{tabular}{|c|c|c|c|c|c|}
\hline Parâmetros & T01 & T02 & T03 & T04 & Média \\
\hline $\begin{array}{c}\text { Consenso } \\
\text { 1a Tentativa }\end{array}$ & $\begin{array}{c}2 / 3 \\
(66,7 \%)\end{array}$ & $\begin{array}{c}1 / 5 \\
(20 \%)\end{array}$ & $\begin{array}{c}2 / 4 \\
(50 \%)\end{array}$ & $\begin{array}{c}2 / 4 \\
(50 \%)\end{array}$ & $\mathbf{4 6 , 6 8 \%}$ \\
\hline Colaboração? & $\mathrm{Sim}$ & $\mathrm{Sim}$ & $\mathrm{Sim}$ & $\mathrm{Sim}$ & - \\
\hline $\begin{array}{c}\text { \% estudantes que } \\
\text { interagiram }\end{array}$ & $93,3 \%$ & $84 \%$ & $93,75 \%$ & $87,5 \%$ & $\mathbf{8 9 , 6 \%}$ \\
\hline Material de apoio & $1 / 3$ & $0 / 5$ & $1 / 4$ & $1 / 4$ & $\mathbf{2 1 \%}$ \\
\hline Tempo suficiente & $(33,3 \%)$ & $(0 \%)$ & $(25 \%)$ & $(25 \%)$ & $\mathbf{2 1}$ \\
\hline & $(66,7 \%)$ & $5 / 5$ & $4 / 4$ & $4 / 4$ & $\mathbf{9 1 , 6 8 \%}$ \\
\hline
\end{tabular}

\begin{tabular}{|c|c|c|c|c|c|}
\hline Parâmetros & T01 & T02 & T03 & T04 & Média \\
\hline $\begin{array}{c}\text { Colaboração? } \\
\text { \% estudantes que } \\
\text { interagiram }\end{array}$ & Sim & Sim & Sim & Sim & - \\
\hline Material de apoio & $\begin{array}{c}1 / 3 \\
(33,3 \%)\end{array}$ & $\begin{array}{c}100 \% \\
(20 \%)\end{array}$ & $\begin{array}{c}100 \% \\
(0 \%)\end{array}$ & $\begin{array}{c}100 \% \\
(25 \%)\end{array}$ & $\mathbf{9 8 , 3} \%$ \\
\hline Tempo suficiente & $0 / 3$ & $0 / 5$ & $1 / 4$ & $0 / 4$ & $\mathbf{1 9}, \mathbf{5 8} \%$ \\
\hline & $(0 \%)$ & $(0 \%)$ & $(25 \%)$ & $(0 \%)$ & $\mathbf{6 , 2 5 \%}$ \\
\hline
\end{tabular}

A Tabela 2 mostra o desempenho geral dos Grupos de Teste na estratégia de Regulação Mútua. É possível observar que $75,43 \%$ dos participantes demonstraram atenção à atividade em algum momento dessa etapa do experimento. Ressalta-se ainda que $64,18 \%$ não obedeceram ao protocolo e interromperam a contribuição do participante da vez a fim de auxiliá-lo, bem como o próprio participante recorreu aos outros objetivando elucidar questões como a lógica utilizada pelo participante anterior no processo, verificação de escrita ou mesmo consulta sobre como resolver a questão. Em sua maioria, cerca de $87,9 \%$ dos participantes mostraram-se motivados em sua respectiva tentativa para resolver a questão.

Tabela 2: Resultados obtidos sobre a estratégia de Regulação Mútua.

\begin{tabular}{|c|c|c|c|c|c|}
\hline Parâmetros & T01 & T02 & T03 & T04 & Média \\
\hline $\begin{array}{l}\text { Atenção } \\
\text { à atividade }\end{array}$ & $\begin{array}{c}2 / 3 \\
(66,7 \%)\end{array}$ & $\begin{array}{c}3 / 5 \\
(60 \%)\end{array}$ & $\begin{array}{c}4 / 4 \\
(100 \%)\end{array}$ & $\begin{array}{c}3 / 4 \\
(75 \%)\end{array}$ & $75,43 \%$ \\
\hline Colaboração? & Sim & Sim & Sim & Sim & - \\
\hline Interrupção & $\begin{array}{c}2 / 3 \\
(66,7 \%)\end{array}$ & $\begin{array}{c}2 / 5 \\
(40 \%)\end{array}$ & $\begin{array}{c}3 / 4 \\
(75 \%)\end{array}$ & $\begin{array}{c}3 / 4 \\
(75 \%)\end{array}$ & $64,18 \%$ \\
\hline Motivação? & $\begin{array}{c}2 / 3 \\
(66,7 \%)\end{array}$ & $\begin{array}{c}5 / 5 \\
(100 \%)\end{array}$ & $\begin{array}{c}4 / 4 \\
(100 \%)\end{array}$ & $\begin{array}{c}4 / 4 \\
(85 \%)\end{array}$ & $87,93 \%$ \\
\hline Abstenção? & Não & Não & Não & $\operatorname{Sim}(3)$ & - \\
\hline $\begin{array}{c}\text { Material de } \\
\text { apoio }\end{array}$ & $\begin{array}{c}1 / 3 \\
(33,3 \%)\end{array}$ & $\begin{array}{l}0 / 5 \\
(0 \%)\end{array}$ & $\begin{array}{l}0 / 4 \\
(0 \%)\end{array}$ & $\begin{array}{c}0 / 4 \\
(0 \%)\end{array}$ & $8,33 \%$ \\
\hline Ciclos (média) & $N / d$ & 1,4 & 1,5 & 1 & 1,3 \\
\hline
\end{tabular}

Analisando-se os dados coletados providos pelos participantes através dos questionários qualitativos, observou-se que há consonância com as informações coletadas pelas observações dos Monitores durante o experimento. A Tabela 3, baseada na proposta de Mcclelland (1976) através da utilização de afirmações positivas e negativas e uso da escala de Likert (Discordo Completamente, Discordo Parcialmente, Indiferente, Concordo Parcialmente, Concordo Completamente), traz uma série de sentenças com o intuito de mensurar qualitativamente as estratégias de colaboração do ponto de vista dos participantes. Nela, é possível observar que $46,1 \%$ dos estudantes se sentiram confiantes ao utilizar as estratégias enquanto que 44,7\% mostraram-se indiferentes. Em contrapartida, 44,7\% concordaram completamente que não há necessidade de possuir muito conhecimento prévio para fazer uso das estratégias com confiança.

É possível observar ainda que $75 \%$ dos participantes sentiram-se estimulados a interagir com os colegas através do uso das estratégias, bem como $82,9 \%$ afirmaram gostar da interação através das estratégias, demonstrando um expressivo sinal sobre a efetividade das mesmas. Por fim, $71 \%$ dos participantes sentiram que o aprendizado foi 
VI Congresso Brasileiro de Informática na Educação (CBIE 2017)

Anais do XXVIII Simpósio Brasileiro de Informática na Educação (SBIE 2017)

efetivo utilizando as estratégias de colaboração, assim como, 52,6\% concorda fortemente que houve evolução no aprendizado ao utilizar as estratégias.

Tabela 3: Resultado da Avaliação Qualitativa das Estratégias pela Visão dos Participantes (Formatação Condicional).

\begin{tabular}{|l|c|c|c|c|c|}
\hline \multicolumn{1}{|c|}{ Descrição } & $\begin{array}{c}\text { DISCORDO } \\
\text { COMPLETAMENTE }\end{array}$ & $\begin{array}{c}\text { DISCORDO } \\
\text { PARCIALMENTE }\end{array}$ & INDIFERENTE & $\begin{array}{c}\text { CONCORDO } \\
\text { PARCIALMENTE }\end{array}$ & $\begin{array}{c}\text { CONCORDO } \\
\text { COMPLTAMENTE }\end{array}$ \\
\hline $\begin{array}{l}\text { Eu gostaria de usar uma } \\
\text { ferramenta com estas estratégias. }\end{array}$ & $1,3 \%$ & $2,6 \%$ & $18,4 \%$ & $38,2 \%$ & $39,5 \%$ \\
\hline $\begin{array}{l}\text { Eu acho que uma ferramenta com } \\
\text { estas estratégias seria complexa. }\end{array}$ & $25,0 \%$ & $26,3 \%$ & $25,0 \%$ & $13,2 \%$ & $10,5 \%$ \\
\hline $\begin{array}{l}\text { Eu penso que as estratégias são de } \\
\text { fácil entendimento. }\end{array}$ & $2,6 \%$ & $5,3 \%$ & $19,7 \%$ & $36,8 \%$ & $35,5 \%$ \\
\hline $\begin{array}{l}\text { Eu precisaria do apoio de uma } \\
\text { pessoa técnica para ser capaz de } \\
\text { usar uma ferramenta com essas } \\
\text { estratégias. }\end{array}$ & $32,9 \%$ & $23,7 \%$ & $26,3 \%$ & $10,5 \%$ & $6,6 \%$ \\
\hline $\begin{array}{l}\text { Eu acho que havia muita } \\
\text { inconsistência nas estratégias. }\end{array}$ & $38,2 \%$ & $15,8 \%$ & $38,2 \%$ & $6,6 \%$ & $1,3 \%$ \\
\hline $\begin{array}{l}\text { Imagino que a maioria das pessoas } \\
\text { iria aprender a usar esta ferramenta } \\
\text { com estas estratégias muito } \\
\text { rapidamente. }\end{array}$ & $0,0 \%$ & $13,2 \%$ & $19,7 \%$ & $48,7 \%$ & $18,4 \%$ \\
\hline $\begin{array}{l}\text { Eu acho que uma ferramenta com } \\
\text { estas estratégias seria muito } \\
\text { complicada de usar. }\end{array}$ & $50,0 \%$ & $32,9 \%$ & $11,8 \%$ & $3,9 \%$ & $1,3 \%$ \\
\hline $\begin{array}{l}\text { Eu me senti muito confiante } \\
\text { usando as estratégias. }\end{array}$ & $6,6 \%$ & $2,6 \%$ & $44,7 \%$ & $30,3 \%$ & $15,8 \%$ \\
\hline $\begin{array}{l}\text { Eu precisaria aprender um monte } \\
\text { de coisas antes que eu pudesse } \\
\text { começar a usar uma ferramenta } \\
\text { com estas estratégias. }\end{array}$ & $44,7 \%$ & $17,1 \%$ & $10,5 \%$ & $21,1 \%$ & $6,6 \%$ \\
\hline $\begin{array}{l}\text { Eu me senti estimulado a interagir } \\
\text { com meus colegas através das } \\
\text { estratégias. }\end{array}$ & $5,3 \%$ & $2,6 \%$ & $17,1 \%$ & $21,1 \%$ & $53,9 \%$ \\
\hline $\begin{array}{l}\text { Eu não gostei de interagir } \\
\text { utilizando as estratégias. }\end{array}$ & $55,3 \%$ & $27,6 \%$ & $9,2 \%$ & $3,9 \%$ & $3,9 \%$ \\
\hline
\end{tabular}

Os participantes apontaram ainda a estratégia de colaboração "argumentação" como a mais interessante e efetiva, pois o ato de primeiro resolver a questão sozinho e depois confrontar os resultados com o grupo traz a possibilidade de conhecer novas visões sobre a resolução de um mesmo problema ampliando o campo de conhecimento do estudante. As estratégias de colaboração "explanação" e "regulação mútua" aparentemente acabaram empatando na preferência dos usuários.

\section{Considerações Finais}

Através do experimento com os participantes, pode-se evidenciar que as estratégias de colaboração possuem robustez para estimular os alunos sendo assim um elegível aliado do ensino a distância no combate à evasão acadêmica anteriormente exposta por investir nos conceitos de flexibilidade e independência defendidos pela $\mathrm{EaD}$ e trazer consigo a possibilidade de interação social, que é um dos principais problemas observados nesse estudo. As perspectivas de futuro deste trabalho concernem na implementação do CollabEduc embarcado com as estratégias de colaboração definidas, bem como na avaliação longitudinal para verificar o impacto do uso do mesmo sobre o desempenho dos alunos.

\section{Referências}

ABED. Censo EaD.br: relatório analítico da aprendizagem a distância no Brasil 2013. Associação Brasileira de Educação a Distância, 2014. 
VI Congresso Brasileiro de Informática na Educação (CBIE 2017)

Anais do XXVIII Simpósio Brasileiro de Informática na Educação (SBIE 2017)

ANDREWS, R. The importance of argument in education. Institute of Education, Uni. of London, 2009.

ARONSON, E; BLANEY, N.; STEPHIN, C.; SIKES, J.; SNAPP, M. The jigsaw classroom. Beverly Hills, CA: Sage Publications, 1978.

BLOM, J.; VERMA, H.; LI, N; SKEVI, A.; DILLENBOURG, P. MOOCs are More Social than You Believe. eLearning Papers, Barcelona, Spain, v. 33, p. 1-3, May 2013.

CHARMAZ, K. Constructing Grounded Theory. A Practical Guide Through Qualitative Analysis. London: Sage Publications, 2006.

CHIONG, R.; JOVANOVIC, J. Collaborative learning in online study groups: An evolutionary game theory perspective. Journal of Information Technology Education: Research, v.11, n. 1, p. 81-101, 2012.

COSTA SEGUNDO, R. M. Athus: um framework para o desenvolvimento de jogos para TV Digital utilizando Ginga. João Pessoa: UFPB, 2011.

COSTA, A. Desenvolver a capacidade de argumentação dos estudantes: um objectivo pedagógico fundamental. Revista Iberoamericana de Educación, v. 46, n. 5, p. 2, 2008.

CUNHA, F. O. M.; SIEBRA, C. D. A. Especificação de Perfis de Grupos para Suporte à Formação de Grupos Colaborativos na EAD. Anais dos Workshops do CBIE 2015, Maceió, 26 out. 2015. 625-634.

DILLENBOURG, P.; BAKER, M.; BLAYE, A.; O'MALLEY, C. The evolution of research on collaborative learning. Learning in Humans and Machine: Towards an interdisciplinary learning science., p. 189-211, 1996.

DILLENBOURG, P.; HONG, F. The mechanics of CSCL macro scripts. ComputerSupported Collaborative Learning, v. 3, p. 5-23, 2008.

FERREIRA, R. M. C.; FELIZOLA, M. P. M. "Teoria Fundamentada em Dados". Uma experiência metodológica. Revista Latinoamericana de Metodología de la Investigación Social, Argentina, v. 3, n. 2, p. 7-19, Abril-Septiembre 2012.

LIMA, E. F. C. Proposta de Objetos de Aprendizagem para Uso de Estratégias de Colaboração em Pequenos Grupos de Estudo. João Pessoa: UFPB, 2016.

LUCA, G. G. D.; KUBO, O. M. O argumentar como objetivo de ensino a ser desenvolvido em diferentes disciplinas e desde níveis básicos do ensino formal. Psicol. educ., São Paulo, n. 33, p. 153-160, 2011.

MAIA, C. Is there a future for distance learning in Brazil? Proceedings of the 6th International Conference on Networked Learning 8. [S.1.]: [s.n.]. 2008. p. 843-844.

MCCLELLAND, J. A. G. Técnica de questionário para pesquisa. Revista Brasileira de, 1976.

MELLINGSÆTER, M. S. Meaning Making Through Use of Interactive Whiteboards During Physics Group Work: A Case Study from Engineering Education. [S.1.]: Norges teknisk-naturvitenskapelige universitet, Fakultet for naturvitenskap og teknologi, Institutt for fysikk, 2014.

MULLER MIRZA, N.; PERRET-CLERMONT, A.-N. Introduction. In: MULLER MIRZA, N. A. P.-C. A.-N. Argumentation and Education. [S.1.]: Springer US, 2009. p. 1-5.

PIAGET, J. W. F. A equilibração das Estruturas Cognitivas-Problema Central do Desenvolvimento. Rio de Janeiro: Zahar Editores, 1976.

PIAGET, J. W. F. O desenvolvimento do pensamento: equilibração das estruturas cognitivas. Lisboa: Dom Quixote, 1977.

SHERRY, L. Issues in distance learning. International journal of educational telecommunications, v. 1, n. 4, p. 337-365, 1995.

SIEMENS, G. Connectivism: A Learning Theory for the Digital Age, 2004.

VYGOTSKY, L. S. Pensamento e Linguagem. Lisboa: Ed. Antídoto, v. 42, 1979. 